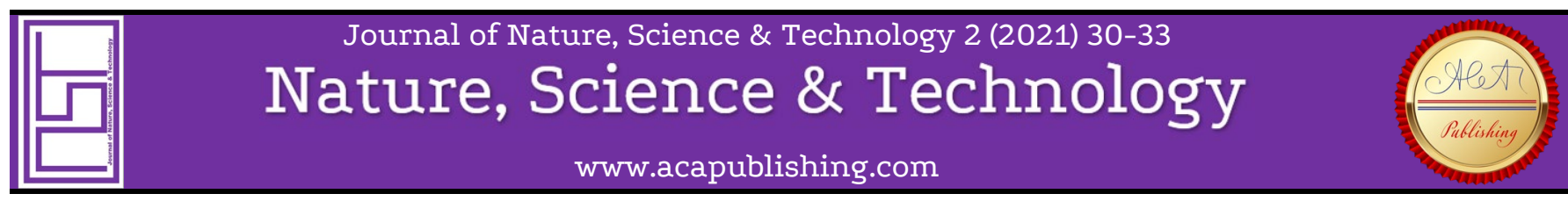

Research Article

\title{
The effect of play-wear on surface roughness of steel music strings
}

\author{
Ferhat Bulbul ${ }^{*},{ }^{1}$ Erdal Sönmez ${ }^{2}$, Kadri Vefa Ezirmik ${ }^{3}$ \\ ${ }^{1}$ Erzurum Technical University, Mechanical Engineering Department, Erzurum, Turkey \\ ${ }^{2}$ Ataturk University Advanced Materials Research Laboratory, Department of Nanoscience \& Nanoengineering, Graduate \\ School of Natural and Applied Sciences, Erzurum, Turkey \\ ${ }^{3}$ Ataturk University, Department of Metallurgical and Materials Engineering, Erzurum, Turkey
}

\section{Keywords}

Wear,

Stick-slip,

Steel,

Stringed musical instrument,

Surface roughness

\begin{abstract}
The play-wear tests on the strings from high carbon steel were realized by using a long necked bağlama stringed instrument. These tests were carried out by reciprocating with the finger on the sound table for different times. The stick-slip wear and striking effects were observed on the strings by AFM. A noticeable increase was detected in the values of surface roughness obtained from $\mathrm{Do}, \mathrm{Fa}$, and $\mathrm{Si}$ pitch-strings and the chest region-string depending on the beat amount. Hence, it attracts attention that the roughness values decrease while it is passed from 1000 to 3000 beats and from Do pitch to Fa concerning beat amount and pitches. However, such an increase was not detected in the Si pitch-string and the chest region-string, and whilst passing from 3000 to 6000 beats, on the contrary, the average surface roughness values showed a tendency to increase.
\end{abstract}

\section{Introduction}

As string core materials for stringed musical instruments, solid steel, rope or cable core steel, synthetic, and gut, whose tonal properties are distinctly different, are commonly used. According to others, steel strings produce larger, livelier and brighter volumes of sound and are cheaper [1]. The strings of stringed instruments are subjected to mechanical (tension, pressure, wear), thermal (temperature), chemical (humidity, dust) and optical (light) effects, and failures and spoils in time, the timbre, then they became useless [2], therefore most string players have to replace their strings in a time.

The experimental works about wear and other negative effects on the tonal peculiarity of strings are too little. However, several theoretical and experimental studies on music strings have been carried out [317]. The present study focuses on the surface roughness of the playwear on uncoated steel strings due to playing. The play-wear on the bağlama (traditional Turkish musical stringed instrument) strings (high carbon steel) was realized by reciprocating with the finger on the regions of Do (C) major and contrariwise scales at andante moderato speed of 100 beat per minute for 10,30 , and 60 minutes. The worn regions from the strings samples were monitored via AFM.

\section{Experimental details}

\subsection{Medium}

The play-wear tests were administered in a quiet room (Figure 1.) where the relative humidity, illumination, temperature, and sound level were at $\% 46 \mathrm{RH}, 0.18 \mathrm{lux}, 25^{\circ} \mathrm{C}$, and $20 \mathrm{~dB}$, respectively.
Test room

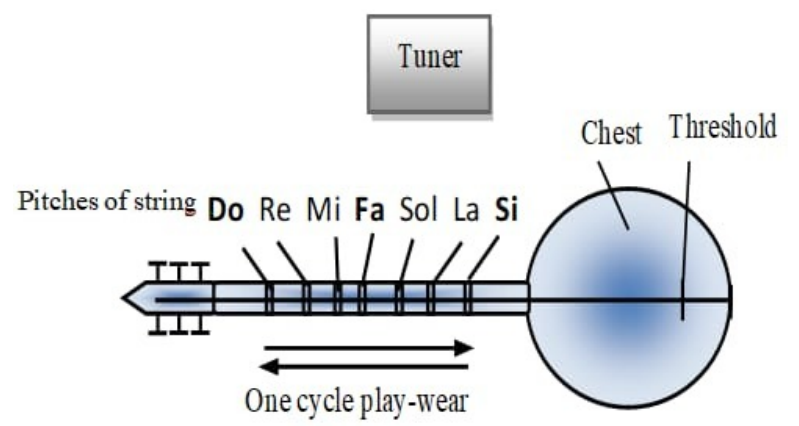

Figure 1. Test medium

\subsection{Used strings}

As a string wire for bağlama instrument, ASTM A228-high carbon steel music wire [18] ( $\varnothing 20$ diameter) was used. Figure 2 . shows a typical AFM photo of an unworn string. 


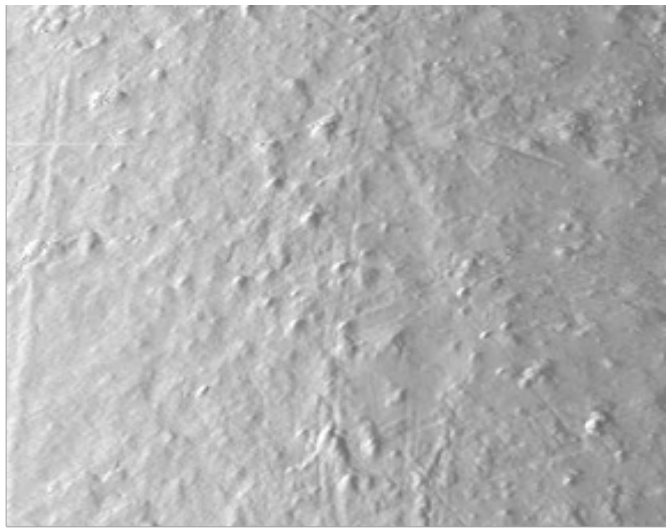

Figure 2. A typical AFM photograph of unworn string

\subsection{The properties and tuning of used stringed instrument}

A long necked bağlama as a stringed instrument picked with a polymer plectrum, was used in play-wear tests (Figure 3.). The accord arrangement was Bozuk or Kara düzen, (tuning or accord arrangement used for commonly long-necked "bağlama") was tuned to A3 Note by a tuner and NI LabVIEW Signal Express Tektronix Edition program connected to the oscilloscope. Accordingly, the total tensional force of the strings on the bağlama was corresponded to $4 \mathrm{~kg}$ [15]. Figure 4. shows a zoomed frequency curve at A3 note (third octave La sound) for unworn string. The frequency curve means that the tuning is very accurate, and it is taken as the base frequency value before all wear tests are performed. A fret defines a raised portion on the neck of a stringed instrument. The Fretboard is the front side of a bağlama neck which contains the frets. When the string is stopped by a finger at the fret, it will produce a note of the desired pitch. The frets of bağlama shown in Figure 3. was from nylon gut. The material of fre is not important parameter for determining wear behavior and damage. Even if each of the frets is a hindrance to the reciprocating movement of the finger, this total effect will be statistically the same in every region of neck.

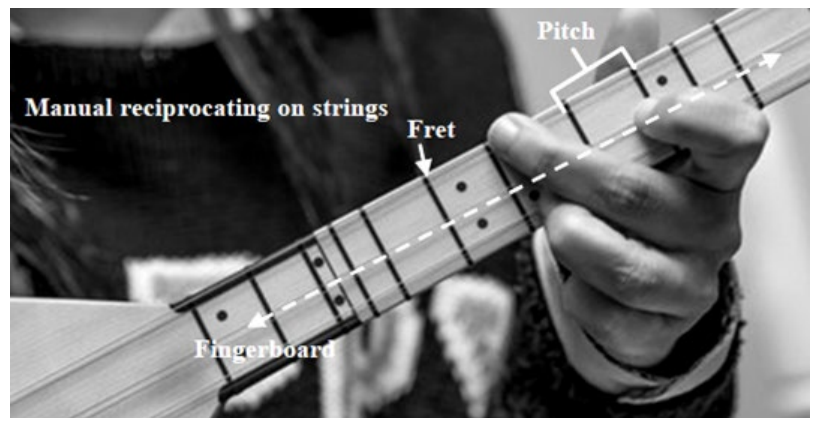

Figure 3. Pitches and frets on the fingerboards of a bağlama

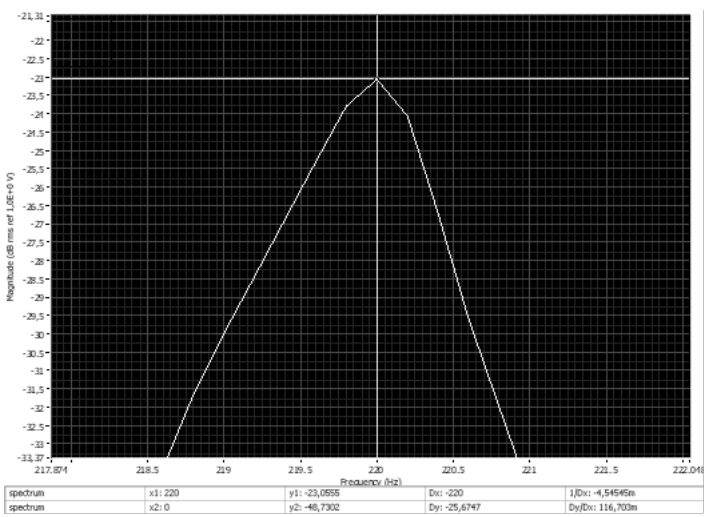

Figure 4. A typical frequency curve belongs to an unfrayed string obtained at $\mathrm{A} 3$ note

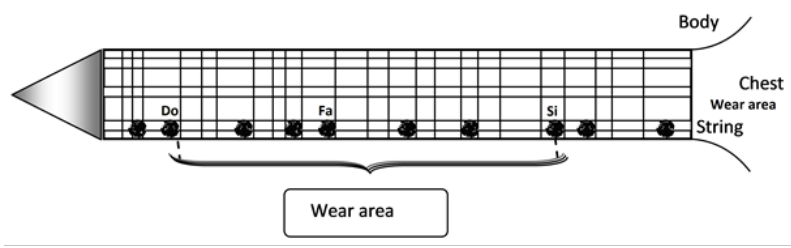

Figure 5. The play-worn regions and beat style with the plectrum

\subsection{Play-wear tests}

Each note has a specific duration. In music notation, a note value indicates the relative duration of a note. There are many different scales, and the major scale or Ionian scale includes 7 notes (Do, Re, $\mathrm{Mi}$, $\mathrm{Fa}$, Sol, $\mathrm{La}$, Ti (or $\mathrm{Si}$ ), that is C-D-E-F-G-A-B. To precisely convey the speed of music, metronome indication was used. The reciprocating with the finger on a totally 14 pitches at once forth Do major scale was carried out on the steel strings as shown in Figure 5. One cycle of reciprocation by finger was completed on the strings at andante moderato speed of 100 beat per minute via the tuner, for durations of 10,30 and 60 minutes. The chest regions were also stroked with the plectrum while reciprocating by finger on the neck is realized. After the worn strings were detaching, the images were taken from the worn regions corresponding to Do (C note), Fa (F note), and Si (H note).

\subsection{AFM studies}

AFM views of worn string surfaces were recorded before and after play-wear using Nanomagnetics Instrument (NMI Image Analyzer) $\mathrm{AFM}$, after the sample string parts corresponded to Do (C note), Fa (F note) and $\mathrm{Si}$ (H note) pitches were embedded on one side of the plastic mold parts. Furthermore, the surface roughness data of the strings were obtained by using AFM Image Analyzer program.

\section{Results and discussion}

After sampling both from $\mathrm{Do}, \mathrm{Fa}$, Si pitches and from the chest regions stroked with the plectrum, AFM photos were obtained from the string parts chosen. It is understood from the images that the straight tracks in different directions show up in the result of the micro-cutting of the string by finger skin and the abrasion by wear particles perhaps from steel string surface.

Figure 6 shows typical AFM views belong to the steel string samples from $\mathrm{Do}, \mathrm{Fa}$, and $\mathrm{Si}$ pitches, respectively. Figure 6.a shows that the flakes on the string that have been worn for 1000 beats. It attracts attention that, as seen in Figure 6.b, the wear process tends to exhibit a preferred orientation along the sliding direction. Furthermore, the highest abrasive effects on Si pitch-string showed oneself with an increase in wear debris and deep track formation. Consequently, from AFM views, it is evaluated that a variety of wear actions- from ploughing abrasion to galling, gouging, chip formation, and dragging, clustering, and separations in the form of flakes - arise due to the beat amount.

Stick-slip has a considerable effect on the formation of wear mechanisms on the string surfaces. Namely, the stick mechanism has a more pronounced effect than the slip-on Do and Si note-strings, while the slip mechanism on Fa pitch-string is more effective. It is considered that the wear tracks taken from Fa pitch-string are more stable and smoother owing to what is mentioned above. Furthermore, the wear damage of $\mathrm{Si}$ pitch-string is the most drastic of all. The possible causes of this would be that, since Si pitch is only a knot point and more intense damages at the "Si" position most likely arises because of more sliding between the string and fret at this position, which is nearer the center part of the open string length, within others, $\mathrm{Si}$ is a note sounding at the highest frequency in addition to the dominant stick mechanism together with slip developing. In addition to this, the string might have been revealing more acoustic energy since it vibrates more at high frequencies. Still, more studies have to be carried out to prove that. Spiny lobsters stick and slip phenomenon, suggested by Patek $[19,20]$, can be simulated to bağlama stringed instrument. The stick-slipped string acts as a file, and the finger contact on the string also causes tactile wear. 


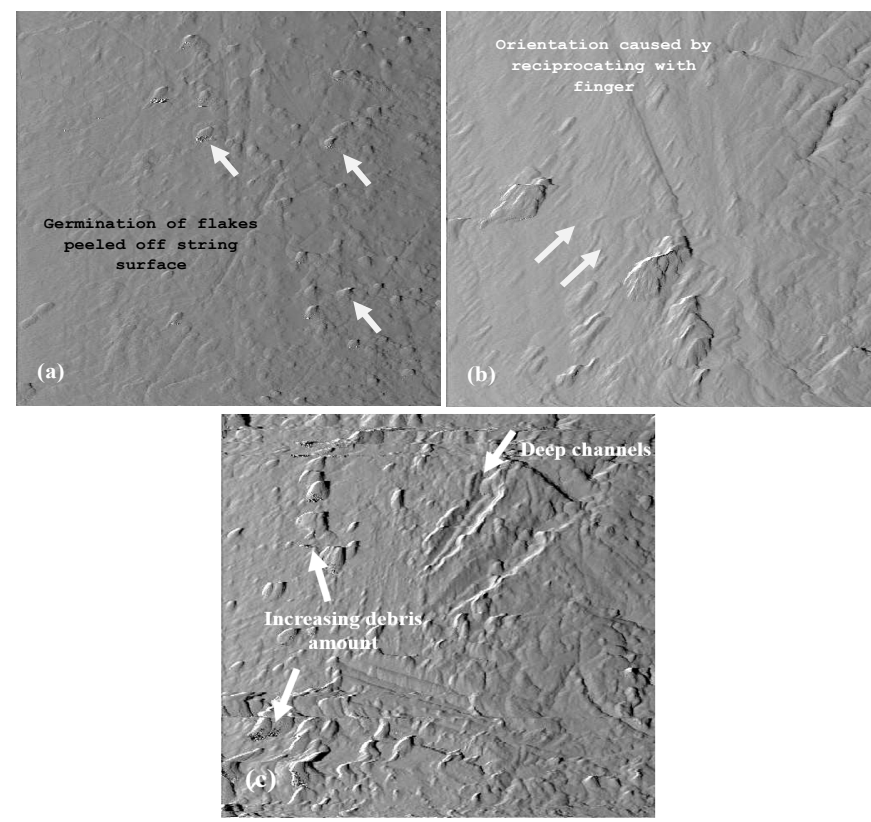

Figure 6. The sample AFM views obtained from (a) Do (play-worn for 1000 beats), (b) Fa (play-worn for 3000 beats), and Si (play-worn for 6000 beats) pitches

Repetitive impacts involve an act that brings the string to damage, even though thermoplastic plectrum is softer than steel. Repetition phenomenon is more important for the wear modes such as pitting, fretting, spalling, impact, brinelling, solid particle impingement, liquid impingement, cavitation, slurry erosion, and partly polishing. The AFM views have demonstrated this condition. The wear emerging with plectrum is caused by the repetitive impacts on the strings of the chest (face part) region of bağlama.

Gouging and chip formations occur with strokes of the plectrum on the string, which comes out due to repetitive impacts in opposite directions. Thus, the vibration caused by the striking of polymer plectrum repeatedly on the same chest region causes small chips to be broken off from the string surface. Therefore, it is seen the wear on the chest string is more intense than that of the neck region.

The wear type commonly seen in stringed instruments is defined as stick-slip wear, and many scientists have studied on and tried to describe it with some theoretical models [21-39]. However, it has been seen that each model can be applied to only to a specific type of a real system.

Figure 7. shows Ra (average surface roughness), Rq (root mean square), Surface Skewness (Sk), and Surface Kurtosis (K) values obtained via AFM, controlled by Nanomagnetics Instrument (NMI Image Analyzer) program. An explanation is graphically given in Figure 7 so that the average surface roughness-beat amount relation would be described better. When Figure 7 is examined, an obvious increase would be detected in the surface roughness values obtained from $\mathrm{Do}, \mathrm{Fa}$, and $\mathrm{Si}$ pitch-strings and the chest region-string depending on the beat amount. Hence, it attracts attention that the roughness values decrease while it is passed from 1000 to 3000 beats and from Do pitch to $\mathrm{Fa}$ with respect to beat amount and pitches. However, such an increase was not detected in the Si pitch-string and the chest regionstring, and whilst passing from 3000 to 6000 beats, on the contrary, the average surface roughness values showed a tendency to increase.

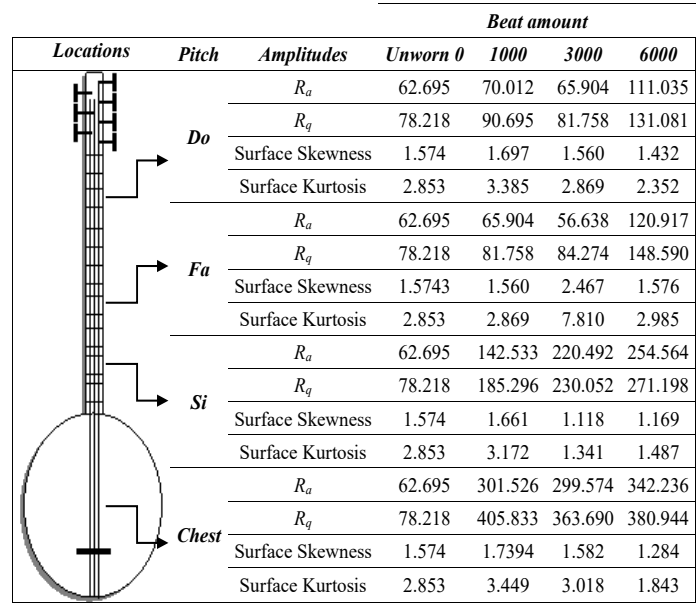

Figure 7. Surface roughness amplitudes and relationships on the strings depending on the beat number and notes

- Do वFa $\square$ Si $\square$ Chest

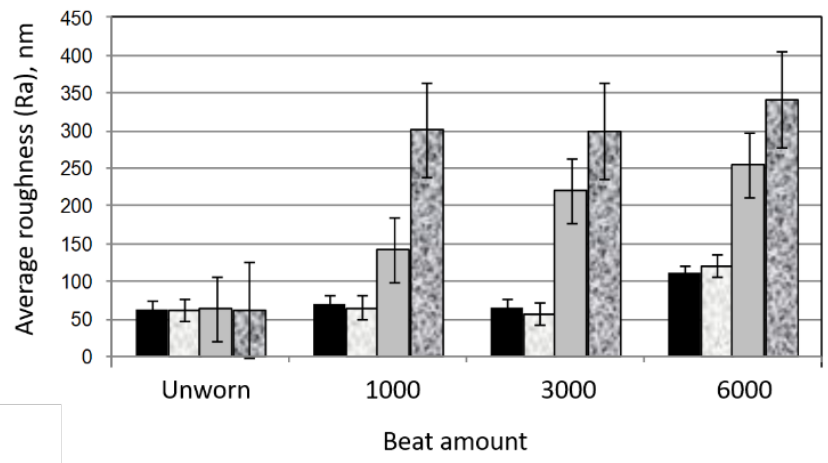

Figure 8. Surface roughness-beat amount relations

As the beat amount increases, surface roughness increased accordingly (Figure 8). Hence, as a result of stick-slip abrasion, the section of the string reduced. However, this reduction was not homogeneous for the whole string with regard to the nature of the abrasion mechanism. Therefore, in the abrasive last products the effect of abrasion heightened causing an increase in the surface roughness. Such an increase caused non-uniformity of the section of the string, further leading to variable section areas.

\section{Conclusion}

The level of wear damage of strings increases depending on the beat amount. That is to say, the play-wear damage was note-worthy even if the wear or the beat amount was not very high. Increasing beat number generally increases the surface roughness of music strings. It is seen that this effect is more prominent in high-pitched sounds with high frequency and more destructive in the chest region. As understood from the results, the investigations on wear-acoustic relationships will be undertaken the role of a router in terms of the production of the strings having long-life and better properties. Furthermore, each of the data to be obtained on strings will also be a considerable scientific resource to be used for all of stringed instruments. 


\section{Acknowledgement}

This research is a part of the TUBITAK (The Scientific and Technical Research Council of Turkey) project supported by grant no: 107M647. The author would like to thank the TUBITAK for funding the project. Besides, the authors thank Atatürk University (Grant no: 2007/151) for providing partly financial supports.

\section{Declaration of Conflict of Interests}

The authors declares that there is no conflict of interest. They have no known competing financial interests or personal relationships that could have appeared to influence the work reported in this paper.

\section{References}

[1.] http://www.knilling.com/pdf/KnillingRefGuide.pdf

[2.] N. Kalender, Instrument Curing and Repairing, J.U.U. Teac. Fac., 14 (2001) 159-166.

[3.] J. Woodhouse, P.M. Galluzzo, The bowed string as we know it today, Acta. Acust. United. Ac., 20 (2004) 579-589.

[4.] L.K. Kitto, Frontiers in Education, 33rd ASEE/IEEE Frontiers in Education Conference, vol. 3, Session 4A-11, November 5-8, Boulder, Colorado, 2003.

[5.] S.F. Liang, W.Y.S. Alvin, Modeling and analysis of acoustic musical strings by using kelly-lochbaum lattice networks, J. Inf. Sci. Eng., 20 (2004) 1161-1182.

[6.] E. Jansson, Acoustics for Violin and Guitar Makers, Chapter IV, Properties of the Violin and Guitar String, fourth ed., 2002.

[7.] http://real.uwaterloo.ca/ sbirkett/iron\%20wire.pdf

[8.] S. Birkett, The Physics of Musical Instruments, second edi., Springer, 1998.

[9.] F. Hellwig, Strings and stringing: contemporary documents, Galpin. Soc. J., 29 (1976) 91-104.

[10.] B.O. Küçükyildirim, M.I. Akdoğan, C. Ertek, The investigation of wear and corrosion behavior of electro-guitar strings depending on playing time, 11th International Materials Symposium, Denizli, Turkey, 2006.

[11.] A.V. Olver, D. Wilson, P.S.J. Crofton, Investigation of service failures of steel music wire, Eng. Fail. Anal., 14 (2007) 224-1232.

[12.] R. Inta, The Acoustics of the Steel String Guitar, Ph.D. Thesis, School of Physics, The University of New South Wales Sydney, 2007.

[13.] D.R. Lapp, The Physics of Music and Musical Instruments, Fellow Wright Center for Innovative Science Education Tufts University Medford, Massachusetts David R. Lapp, Fellow Wright Center for Innovative Science Education Tufts University Medford, Massachusetts, 2004.

[14.] H. Wright, The Acoustics and Physcoacoustics of Guitar, Ph.D. Thesis, Department of Physics and Astronomy, University of Wales, College of Cardiff, 1996.

[15.] http://www.cs.helsinki.fi/u/wikla/mus/NewScalc/

[16.] A. Vurma, J. Ross, J. Interd. Music S. Timbre-induced pitch deviations of musical sounds, 11 (2007) 33-50.

[17.] D. Lunney, R. Morrison, Auditory Presentation of Experimental Data, In: E. Farrell, ed., op cit., 1990, pp. 140-146.

[18.] ASTM committee A1 on steel stainless steel and related alloys, standard specification for steel wire, 2000, Music Spring Quality, ASTMA228/A 228M - 90, ASTM.

[19.] S. N. Patek, Spiny lobsters stick and slip to make sound, Nature. 411 (2001) 153-154.

[20.] S. N. Patek, J. E. Baio, The acoustic mechanics of stick-slip friction in the California spiny lobster (Panulirus interruptus), J. Exp. Biol. 210 (2007) 3538-3546.

[21.] A. D. Berman, W. A. Ducker, J. N. Israelachvili, Origin and characterization of different stick-slip friction mechanisms, Langmuir, 12 (1996) 4559-4563.

[22.] T. Baumberger, F. Heslot, B. Perrin, Crossover from creep to inertial motion in friction dynamics, Nature, 367 (1994) 544.

[23.] F. Heslot, T. Baumberger, B. Perrin, Creep, stick-slip, and dryfriction dynamics: Experiments and a heuristic model, Phys. Rev E. 49 (1994) 4973-4988.

[24.] J. B. Sampson, F. Morgan, D. W. Reed, M. Muskat, Friction behavior during the slip portion of the stick-slip process, J. Appl. Phys. 14 (1943) 689.
[25.] F. Heymann, E. Rabinowicz, B. Rightmire, Friction apparatus for very low-speed sliding studies, Rev. Sci. Instr. 26 (1954) 56-58.

[26.] E. Rabinowicz, The intrinsic variables effecting stick-slip process, Proceedings of The Physical Society, Soc. A-Math Phy. 71 (1958) 668-673.

[27.] J. H. Dieterich, Time dependent friction and the mechanics of stick-slip, Pure Appl. Geophys. 116 (1978) 790-806.

[28.] J. H. Dieterich, Modelling of rock friction, Experimental results and constitutive equations, J. Geophys, Res. 84 (1979) 2161-2168.

[29.] H. Yoshizawa, Y. L. Chen, J. J. Israelachvili, Fundamental mechanisms and interfacial friction, Relation between adhesion and friction, Phys. Chem. 9716 (1993) 4128-4140.

[30.] P. A. Thompson, M. O. Robbins, Origin of stick-slip motion in boundary lubrication, Science. 250 (1990) 792-794.

[31.] P. A. Thompson, M. O. Robbins, Critical velocity of stick-slip motion, Science. 253 (1991) 916.

[32.] J. N. Israelachvili, P. M. McGuiggan, Adhesion and short-range forces between surfaces I - New apparatus for surfaces force measurement, J. Mater. Res. 5 (10) (1990) 2232-2243.

[33.] P. A. Thompson, G. S. Grest, M. O. Robbins, Phase transitions and universal dynamics in confined films, Phys. Rev. Lett. 68 (1992) 3448-3451.

[34.] S. Hogmark, S. Jacobson, O. Vingsbo, ASM Handbook on Friction. Lubrication and Wear Technology, Blau. P. Ed. 18 (1992) 176-183.

[35.] M. T. Bengisu, A. Akay, Stick-slip oscillations: dynamic of friction and surface roughness, J. Acoust. Soc. Am. 105 (1999) 194-205.

[36.] A. Akay, Acoustics of friction, J. Acoust. Soc. Am. 111 (2002) 15251548.

[37.] G. G. Adams, Steady sliding of two elastic half-spaces with friction reduction due to interface stick-slip, J. Appl. Mech. 65 (1998) 470475 .

[38.] C. Gao, D. Kuhlmann-Wilsdorf, D. D. Makel, Fundamentals of stick-slip, Wear. 162-164 (1993) 1139-1149.

[39.] C. Gao, Stick-slip motion in boundary lubrication, Tribol T. 38 (1995) 473-477.

\section{How to Cite This Article}

Bulbul, F., Sonmez, E., Ezirmik, K.V., The effect of play-wear on surface roughness of steel music strings, Journal of Nature, Science \& Technology, 2(2021), 30-33.

https://doi.org/10.36937/janset.2021.002.005 